\title{
État de l'art de la science et technologie des systèmes d'éclairage : Economies d'énergie et Développement Durable
}

\author{
Georges ZISSIS \\ Université de Toulouse - LAPLACE, bât 3R2, 118 rte de Narbonne, 31062 Toulouse cedex 9 (georges.zissis@laplace.univ-tlse.fr) \\ Cet article, avant relecture et correction pour la revue J3EA, a fait l'objet d'une communication aux journées 2007 de la section \\ électrotechnique sur le thème Energie et Développement Durable à l'antenne de Bretagne de l'ENS de Cachan les 14 et 15 mars 2007
}

\begin{abstract}
Résumé- L'éclairage électrique a profondément bouleversé notre vie quotidienne. Il serait aujourd'hui inimaginable de s'en passer. On estime aujourd'hui à environ 30 milliards le nombre de lampes électriques en service sur la planète. Elles consomment, chaque année, presque $19 \%$ de la production mondiale de l'électricité (ou bien un peu moins de $2 \%$ de l'énergie fossile utilisée par an). La majeure partie de la lumière est produite, aujourd'hui, par les lampes à décharge électrique. Bien que cette technologie soit maîtrisée, l'efficacité lumineuse des sources et la qualité de leur lumière n'ont pas encore atteint leurs limites: il reste encore beaucoup de place pour l'innovation. La mise au point de meilleures sources de lumière pour l'éclairage est un enjeu scientifique, technologique, économique et environnemental. Ce papier présente les différentes familles des lampes modernes, leurs caractéristiques et surtout les challenges pour le futur.
\end{abstract}

Mots clefs: Sources de Lumière, Eclairage, Energie, Environnement, Développement Durable

\section{INTRODUCTION}

L'Homme a toujours voulu continuer à vivre normalement après la tombée de la nuit; astreint aux rythmes et aux aléas des sources naturelles de rayonnement, il s'est efforcé très rapidement à remplacer les sources de lumière naturelle par des sources artificielles. S'il a, sans doute assez tôt, découvert le feu et donc, sans le savoir, l'incandescence, c'est seulement à la fin du XIX ${ }^{\mathrm{e}}$ siècle qu'il a commencé à maîtriser la production de lumière par luminescence, qu'il connaissait déjà, au moins sous sa forme biologique : les lucioles et les vers luisants. Par ailleurs, la lumière est à l'origine d'une large part de nos facultés mentales: il est donc naturel que nous assimilions "sources de rayonnement" et "sources de lumière". En réalité, une part des applications des "sources de rayonnement" se situe dans l'ultraviolet et dans l'infrarouge. Que demande-t-on à une source de lumière (donc destinée à l'éclairage)? Pour répondre à cette question, quelques connaissances sur l'environnement visuel sont nécessaires (Figure 1).

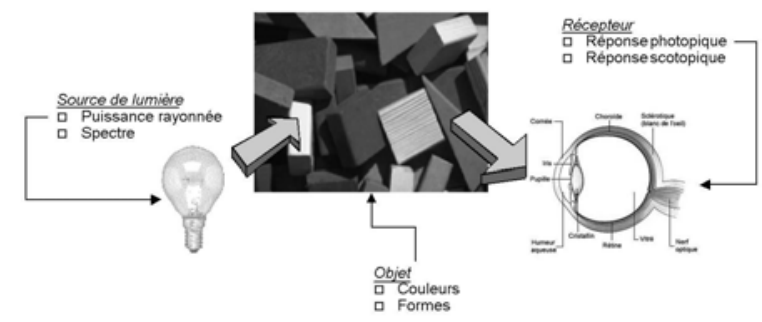

Fig. 1 : Environnement visuel.
Cet environnement résulte de l'interaction de trois éléments : la source, l'objet et le système visuel (récepteur). En pratique l'objectif est de détecter, de définir et de situer, à l'aide du système visuel, l'objet - évènement rendu visible par la source.

Toutefois, "voir" résulte d'une suite d'évènements complexes, dont seuls quelques éléments fondamentaux peuvent être retenus pour caractériser une source de lumière par rapport au système visuel. Les plus importants sont certainement "la sensibilité chromatique" du système visuel et ses "propriétés de perception des sensations colorées". A ces éléments correspondent deux impératifs qui sont aussi deux critères de qualité pour les sources: l'efficacité lumineuse, qui caractérise la quantité de lumière produite par watt consommé et le rendu des couleurs, c'est-à-dire la capacité de produire une lumière qui puisse être considérée comme capable de restituer aux objets leur aspect coloré ordinaire dans des conditions données d'environnement.

En fonction de l'application envisagée, ces deux critères ne sont pas d'une égale importance. Ainsi, pour l'éclairage des routes une grande efficacité sera requise, mais le rendu de couleur sera secondaire. À l'opposé, l'éclairage domestique nécessite un excellent rendu de couleurs, mais on se contente souvent d'une efficacité médiocre.

\section{L'IMPACT ENERGETIQUE, ENVIRONNEMENTAL ET ECONOMIQUE DES LAMPES}

Du point de vue énergétique, l'éclairage consomme plus de 2651 TWh d'énergie électrique par an. Cette quantité représente approximativement $19 \%$ de la production globale d'électricité mondiale $^{1}$ [1] et elle représente une facture annuelle de plus de 200 milliards d'euros pour le consommateur [2]. La situation varie d'un pays à l'autre. Ainsi la consommation énergétique d'un pays occidental pour l'éclairage varie entre $7 \%$ et $15 \%$ de sa propre production énergétique (avec un cas singulier : les USA, où ce pourcentage est de l'ordre de $20 \%$ ).

Plus précisément, la France a consommé, en 1999, 41 TWh pour l'éclairage. Un peu moins de $2 / 3$ de cette énergie (60\% du total) est utilisé par le secteur tertiaire. L'éclairage public et routier consomme $10 \%$ du total, alors que $30 \%$ de l'énergie est absorbée par l'éclairage domestique. Il faut toutefois noter que ce dernier secteur a vu sa consommation

Cette quantité d'électricité représente l'équivalent de 3\% de l'énergie primaire utilisée dans une année sur notre planète (ce calcul considère que l'électricité représente $16 \%$ de la consommation énergétique de la planète). 
multipliée par trois dans une période de deux décennies². Aux Etats-Unis la consommation d'énergie électrique pour l'éclairage atteignait, en l'an 2000, $659 \mathrm{TWh}$ ce qui représente $19 \%$ de l'énergie électrique produite dans le pays (en valeur absolue, cette énergie équivaut à la production d'électricité combinée de la France et de l'Italie). Ici aussi l'éclairage tertiaire domine cette consommation comme le montre le graphique suivant. Il faut toutefois noter que la majeure partie de cette énergie est consommée par des lampes à incandescence $(46 \%)$ sans pour autant produire la majeure partie de la lumière utilisée [3]. La figure 2 compare les situations Française et Américaine. (a)

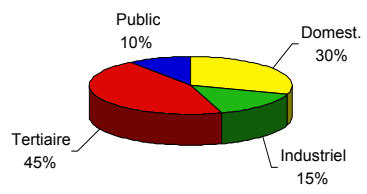

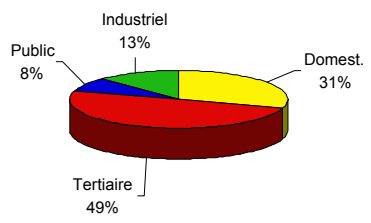

(b)
Fig 2 : Comparatif des consommations énergétiques dédiées à l'éclairage en 1999 (a) en France : 41 TWh et (b) aux USA : 650 TWh

En ce qui concerne maintenant les pays en voie de développement, la situation est différente. L'éclairage représente aujourd'hui la majeure partie de leur consommation électrique : $30 \%$ pour la Tunisie, presque $40 \%$ pour le Madagascar et jusqu'à $86 \%$ pour la Tanzanie (champion toute catégorie dans le domaine). L'éclairage domestique est ici prédominant. Cette situation s'explique facilement compte tenu du fait que la lumière est un besoin intime de l'Homme, et, bien entendu, dès que l'électrification avance la population en profite en installant tout d'abord des lampes. Par ailleurs, dans ces pays, le coût de la lampe est un facteur majeur pour le choix du type de la source de lumière. Ainsi les lampes à incandescences dominent le marché car les lampes «basse consommation» sont souvent inabordables. Encore une fois l'efficacité énergétique est délaissée au profit d'autres considérations...

La production de l'énergie électrique pour satisfaire les besoins en éclairage de l'Homme entraîne inévitablement une pollution de l'environnement. Ainsi, on estime que chaque année quelque 1500 millions de tonnes de $\mathrm{CO}_{2}$ accompagnent cette production d'énergie en contribuant à l'effet de serre ${ }^{3}$.

Par ailleurs, les lampes contiennent des matériaux rares et souvent toxiques $(\mathrm{Hg}, \mathrm{Cd}$, terres rares...), radioactifs ( $\mathrm{Th}$, ${ }^{98} \mathrm{Kr}$ ) et, enfin, leurs ballasts ferromagnétiques contiennent du plomb. Ainsi, à la fin de la vie de la lampe, ces matériaux peuvent se déverser dans la nature. Par exemple, la majorité des lampes, à l'exception des ampoules à incandescence, contiennent du mercure. C'est ainsi, qu'aux USA pour produire chaque année quelque 750 millions de nouveaux tubes fluorescents on utilise 2,5 tonnes de mercure et dans d'autres pays comme la France on collecte par an

\footnotetext{
${ }^{2}$ Consommation d'énergie pour l'éclairage domestique en France : 5 TWh en 1979, 14 TWh en 1999

3 A cette quantité il faut ajouter 400 millions de tonnes de $\mathrm{CO}_{2}$ correspondant à la consommation des lampes à pétrole utilisées encore par 1,5 milliard d'individus
}

approximativement 80 tonnes de déchets contaminés au mercure. Une réglementation européenne, entrée en vigueur le $1^{\mathrm{e}}$ Janvier 1998, impose que les lampes à décharge en fin de vie soient ramenées à des déchets "ultimes» et elles doivent être retraitées avant stockage dans des décharges spéciales (dites de classe «terminale »). Le stockage, dans une décharge de ce type, coûte approximativement 200 euros par tonne de déchets traités. Enfin, concernant les quantités de substances toxiques dans les lampes, il existe depuis 2003 une nouvelle réglementation européenne « RoHS -Removal of Hazardous wastes », que la France a ratifié en 2005 [4]. Cette directive impose des limites à la quantité de mercure présente dans une lampe fluorescente ou à basse consommation.

L'industrie des lampes est en plein développement. La croissance annuelle de ce secteur est de l'ordre de $0,9 \%$. En valeur absolue, ce rythme semble faible, mais il se maintient depuis une bonne vingtaine d'années, ce qui fait de l'industrie des lampes l'une des quatre secteurs industriels avec la plus forte progression sur les deux dernières décennies. Le chiffre d'affaires total pour le secteur industriel des sources de lumière est de 14 milliards d'euros par an, environ ${ }^{4}$. Cependant, l'industrie et le marché des lampes connaissent une vraie spécificité : trois principaux acteurs internationaux concentrent l'essentiel de l'activité : Philips Lighting, General Electric Lighting et SiemensOsram. Ces trois principales sociétés représentent à elles seuls 12 milliards d'euros de chiffres d'affaires... Ce type de structure est qualifié « d'oligopole».

\section{LES FAMILLES DES LAMPES CONTEMPORAINES}

De plus en plus présents dans nos activités, les lampes et l'éclairage ont connu un développement soutenu pendant des siècles. L'utilisation de l'électricité fut une révolution dans le domaine. Aujourd'hui on estime à presque à 33 milliards le nombre de lampes électriques qui fonctionnent sur terre tandis que la production annuelle dépasse les 16 milliards d'unités. Cependant, toutes les sources produisant de la lumière artificielle à partir de l'électricité utilisent toujours les deux procédés de base, l'incandescence et la luminescence identifiée déjà par l'Homme préhistorique.

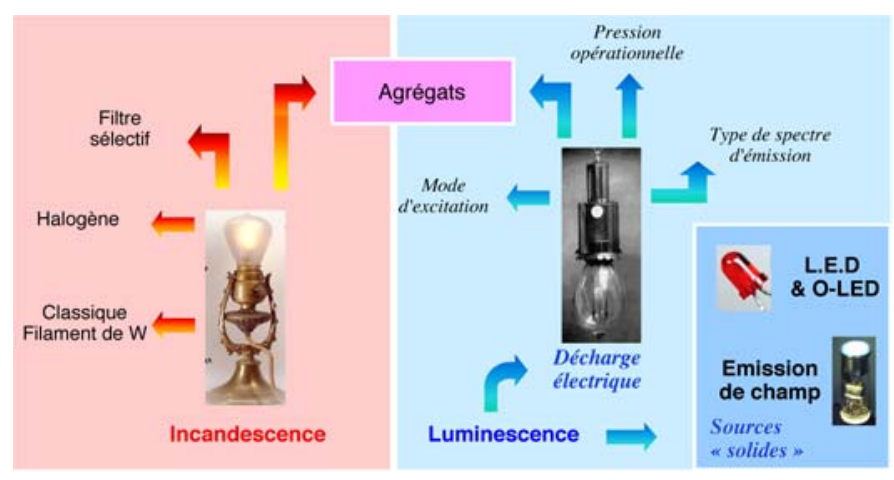

Fig. 3 : Les familles des lampes

La figure 3 présente de façon synthétique les différentes familles de sources susceptibles d'être utilisées pour produire

${ }^{4}$ Ce chiffre d'affaires concerne uniquement les lampes et il n'inclut ni le
secteur du ballast électronique et ni celui des luminaires. Le chiffre
d'affaires global du secteur «éclairage » est probablement 5 fois supérieure. 
de la lumière ou pour assurer des fonctions de signalétique. En regardant de plus près, on y retrouve les familles de sources traditionnelles (ampoules incandescentes et lampes à décharge), mais aussi la famille des diodes électroluminescentes qui a fait son entrée massive dans le monde de l'éclairage depuis plusieurs années maintenant ${ }^{5}$, ainsi que quelques nouveautés comme la lampe à agrégats et la lampe à émission de champs.

Dans les lampes à incandescence (GLS) actuelles, le radiateur (filament) en tungstène est chauffé par effet Joule. C'est un milieu très dense, la densité de puissance y est élevée et l'émission (de surface) présente une forte luminance. Son rayonnement est continu et suit approximativement (dans le visible) la loi des corps « gris » à la température du filament. Cependant, il n'est pas possible d'augmenter beaucoup la température car l'évaporation ponctuelle du filament et sa recristallisation réduisent très vite la durée de vie de la lampe par rupture du filament. Celle-ci dépend essentiellement, à travers ces deux phénomènes, de la tension, des allumages, des vibrations, du champ des températures autour de la lampe... L'autre conséquence de l'évaporation est le noircissement de l'ampoule. On limite cette évaporation par la présence d'un gaz rare qui diminue la vitesse d'évaporation. Toutefois, la grande dimension des ampoules limite la pression du gaz rare à une valeur proche de la pression atmosphérique. Le cycle tungstène - halogène permet, dans les lampes halogènes, de supprimer le dépôt de tungstène sur la paroi. On peut alors réduire la dimension des ampoules et augmenter considérablement la pression du gaz rare ce qui limite l'évaporation. Ce gain sur l'évaporation peut être utilisé directement, en termes de durée de vie, ou être converti, à durée de vie constante, en augmentation de température et donc d'efficacité lumineuse. Ces choix sont faits en fonction de l'utilisation. Pour les lampes halogènes d'éclairage général, on a porté la température de couleur à $3000 \mathrm{~K}$ pour une durée de vie de $2000 \mathrm{~h}$. Dans ces conditions l'efficacité lumineuse est limitée à 20 à $25 \mathrm{~lm} / \mathrm{W}$ ce qui est cependant bien supérieur aux 12 à $14 \mathrm{~lm} / \mathrm{W}$ des lampes à incandescence ordinaires.

Bien que la lampe à décharge électrique réalisée pour la première fois en 1814 par Sir H. Davy et M. Faraday, fût la première application des plasmas, il a fallu attendre plus d'un siècle avant de connaître une réalisation commercialisable. Aujourd'hui, grâce à une meilleure compréhension des processus physiques qui régissent leur fonctionnement, les lampes à décharge se sont considérablement améliorées depuis leur apparition. Une lampe à décharge est constituée d'une enceinte étanche et transparente (ou translucide) qui confine le support gazeux de la décharge tout en laissant échapper le rayonnement que l'on veut utiliser. Cette enceinte, de forme et de dimension très variables, contient un mélange de gaz et/ou de vapeurs métalliques, susceptibles de rayonner dans le domaine des longueurs d'onde désirées. L'excitation du mélange est assurée soit par la circulation d'un courant électrique entre deux électrodes, soit par l'injection dans l'ampoule d'un

\footnotetext{
${ }^{5}$ Les diodes électroluminescentes se déclinent aujourd'hui aux diodes semiconducteurs classiques et à leur version organique connue sous le nom de $\mathrm{O}$ LED.
}

rayonnement électromagnétique haute-fréquence (radiofréquences, micro-ondes,...). Cependant, la caractéristique courant-tension d'une décharge étant négative ou très faiblement positive (cas des lampes haute pression en régime), une impédance est nécessaire pour stabiliser le courant de décharge.

La pression de l'élément actif peut être comprise entre quelques dizaines et quelques millions de Pascal. On a l'habitude de parler de lampe basse ou haute pression (BP et HP respectivement). Ainsi, les lampes « haute pression » fonctionnent à des pressions allant de 1 à plusieurs centaines de bars (200 bars pour la lampe UHP utilisée pour les vidéoprojecteurs, ce qui impose de sévères contraintes à la géométrie de la lampe et de ses électrodes). Dans ces conditions, la luminance du rayonnement approche la luminance $\mathrm{du}$ corps noir à la température $\mathrm{T}_{\mathrm{C}}$ de la zone centrale de la décharge, cette température est typiquement comprise entre $4000 \mathrm{~K}$ et $6000 \mathrm{~K}$. Les efficacités lumineuses atteintes sont actuellement comprises entre $50 \mathrm{~lm} / \mathrm{W}$ et $110 \mathrm{~lm} / \mathrm{W}$. Par ailleurs, nous pouvons contrôler le spectre de la décharge et par conséquent son Indice de Rendu des Couleurs (IRC) en en sélectionnant les corps émissifs (éléments actifs : mercure, sodium, thallium, dysprosium...). La duré de vie de ces produits varie entre 4000 et $20000 \mathrm{~h}$. Les lampes "haute pression" les plus connues sont :

- Les lampes mercure haute pression $\left(\mathrm{T}_{\mathrm{C}} \approx 3000-4500 \mathrm{~K}\right.$, IRC $\approx 50, \quad \eta \approx 50 \mathrm{~lm} / \mathrm{W}$ ), utilisées pour l'éclairage de grandes surfaces (hangars) et pour l'éclairage urbain ;

- Les lampes sodium haute pression $\left(\mathrm{T}_{\mathrm{C}} \approx 2100-2300 \mathrm{~K}\right.$, IRC $\approx 15-60, \eta \approx 50-120 \mathrm{~lm} / \mathrm{W})$, destinées à l'éclairage urbain et routier ainsi qu'à la mise en valeur des monuments ;

- Les lampes à iodures métalliques à enveloppe en silice ou en céramique $\left(\mathrm{T}_{\mathrm{C}} \approx 3000-6000 \mathrm{~K}, \quad \mathrm{IRC} \approx 60-95\right.$, $\eta \approx 50-110 \mathrm{~lm} / \mathrm{W}$ ) pour l'éclairage des stades, des esplanades et pour l'éclairage urbain.

En ce qui concerne les lampes «basse pression », les lampes fluorescentes sont les plus connues de cette famille. Les lampes fluorescentes (appelées à tort « néons », car elles ne contiennent pas ce gaz...) sont aujourd'hui des produits technologiquement matures. C'est par ailleurs le type de lampes à décharge le plus répandu sur terre. La pression dans ce type de lampes est de l'ordre de quelques mbars pour le gaz rare et quelques $\mu$ bars pour l'élément actif (mercure). L'efficacité de ces lampes atteint les $90 \mathrm{~lm} / \mathrm{W}$ et l'IRC dépasse souvent la valeur 80 . La durée de vie de ces produis habituels est comprise entre 6000 et 15000 h. Les lampes dites « à économie d'énergie », ou mieux, «fluo compactes » font partie de cette famille et elles fonctionnent exactement sur le même principe que les lampes fluorescentes linéïques. Leur efficacité est comprise entre 35 et $65 \mathrm{~lm} / \mathrm{W}$ tandis que leur durée de vie varie entre 4000 et $12000 \mathrm{~h}$.

Le principe de fonctionnement simplifié d'une Diode Electroluminescente (LED) est le suivant: l'élément de base de toute diode électroluminescente est un semiconducteur ayant deux régions de conductivité différente (de type $\mathrm{p}$ et $\mathrm{n}$ ) et une région de recombinaison radiative des porteurs « $\mathrm{n} »$ (électrons) et « $\mathrm{p} »$ (trous). Sous l'effet d'une différence de potentiel appliquée entre les deux couches, des électrons pénètrent dans la couche de type $\mathrm{n}$. Ceci équivaut à une injection de trous dans la couche de type p. A l'interface 
de ces deux zones, les électrons et les trous se recombinent en donnent naissance à un photon. À l'état d'équilibre, les porteurs majoritaires de chaque zone diffusent vers l'autre zone : les électrons de la zone « $\mathrm{n}$ » ont tendance à diffuser vers la zone $« p »$, les trous suivent le chemin opposé. Ces mouvements spontanés perturbent la neutralité électrique locale du système et sont à l'origine de l'apparition d'un champ de « charge espace » qui s'oppose, à son tour, à ces mouvements et le système s'équilibre. Par conséquent le nombre de porteurs minoritaires dans chaque zone (électrons dans la zone $p$ et trous dans la zone $n$ ) reste extrêmement faible et la probabilité de recombinaison radiative est quasinulle. En appliquant maintenant à la jonction une tension de polarisation directe $V$, la barrière de potentiel s'abaisse d'une valeur égale à $\mathrm{eV}$. Par conséquent, le courant de diffusion des porteurs majoritaires de chaque coté de la barrière vers la zone opposée augmente par un facteur proportionnel à $\exp \left(e V / k_{B} T\right)$, où $\mathrm{T}$ est la température de la jonction et $k_{B}$ la constante de Boltzmann. Cette augmentation du courant de diffusion déséquilibre le système et abouti à une croissance de la population des porteurs minoritaires dans chaque zone. $\mathrm{Si}$ la tension de polarisation directe est suffisante, la probabilité de recombinaison radiative n'est plus négligeable et des photons sont produits par la jonction. Le principe énoncé ci-dessus correspond à une diode électroluminescente dite « homo-jonction ». Les LEDs classiques utilisent encore aujourd'hui cette structure, mais, les nouvelles diodes de forte intensité utilisent des jonctions bien plus complexes dites «hétéro-jonctions». Elles reposent sur la variation spatiale de la composition du semi-conducteur. Ces structures sont des assemblages des semi-conducteurs de différentes compositions chimiques ayant également des différents « gaps » énergétiques. Aujourd'hui, nous utilisons des hétéro-structures simples ( $\mathrm{SH}$ ) ou doubles (DH). Aujourd'hui, nous savons fabriquer en série des LEDs blanches de puissance (jusqu'à $5 \mathrm{~W}$ ) ayant des efficacités lumineuses de $35-40 \mathrm{~lm} / \mathrm{W}^{6}$ et des durées de vie allant bien au-delà de $50000 \mathrm{~h}^{7}$.

\section{QUELS OBJECTIFS POUR DEMAIN ?}

Répondre à cette question est bien délicat, essayons cependant d'avancer quelques éléments de réponse qui nous permettront aussi de situer les "défis" pour le futur dans le domaine des sources de lumière.

Le premier de ces défis consiste à augmenter l'efficacité lumineuse de la source. L'augmentation de cette efficacité, ne fût-ce que de quelques pour cent, constituerait un progrès important. Des estimations diverses indiquent que, compte tenu de l'augmentation de la demande de l'humanité pour l'éclairage, en utilisant simplement de façon plus raisonnée les sources existantes, nous pouvons économiser entre dix et quinze pour cent de l'énergie consommée pour l'éclairage dans les dix années à venir. Il faut aussi rappeler que l'augmentation de l'efficacité entraînerait une diminution de la pollution de l'environnement liée au fait de la diminution de la production d'énergie nécessaire à l'éclairage. Dans l'état actuel des choses, une augmentation de deux pour cent de

\footnotetext{
${ }^{6}$ Des prototypes allant jusqu'à $120 \mathrm{~lm} / \mathrm{W}$ ont déjà vu le jour.

${ }^{7}$ Cette durée de vie est définie pour la jonction « nue » hors système.
}

l'efficacité énergétique entraînerait une diminution de six à sept millions de tonnes de rejet du $\mathrm{CO}_{2}$ dans l'atmosphère, ce qui correspond à un pour cent de la diminution prévue par les accords de Kyoto sur l'environnement.

Actuellement, malgré tous les progrès de la science et de la technologie dans le domaine des lampes, l'efficacité maximale de lampes qui produisent de la lumière blanche de bonne qualité stagne, depuis les années 70, autour de $120 \pm 10 \mathrm{~lm} / \mathrm{W}$ (Figure 4).

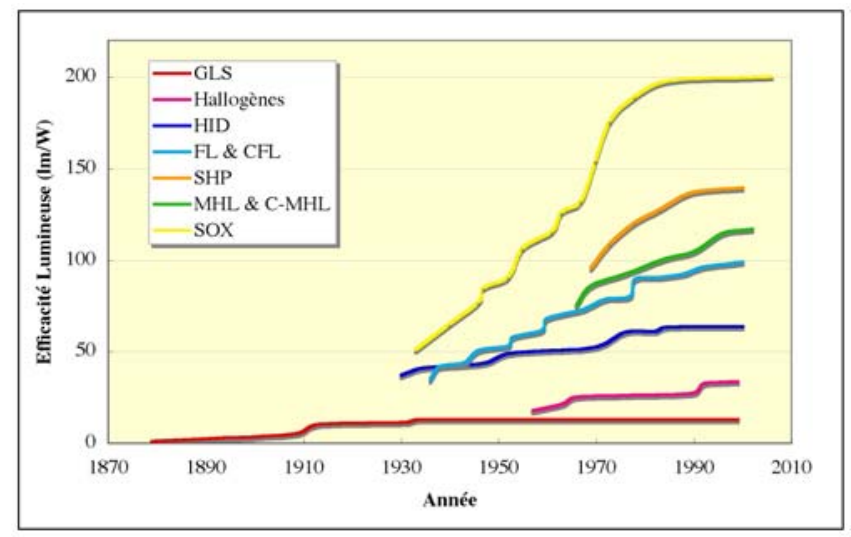

Fig. 4 : Evolution de l'efficacité lumineuse des différentes des sources de lumière (GLS : lampes à incandescence; HID : lampes mercure haute pression ; FL \& CFL : Lampes fluorescentes linéiques et lampes fluorescentes compactes; SHP : lampes sodium haute pression ; MHL et CMHL : lampes à iodures métalliques à enveloppe silice et à enveloppe

céramique ; SOX : Lampes sodium basse pression - elles produisent une lumière jaune monochromatique)

La question qui se pose est alors la suivante : l'industrie des lampes a-t-elle atteint une sorte de "limite thermodynamique?

Pour répondre à cette question essayons de déterminer ce que pourrait être une limite supérieure raisonnable pour l'efficacité lumineuse d'une lampe produisant de la lumière blanche de «bonne qualité» avec une température de couleur, $T_{C}$, comprise entre $3000 \mathrm{~K}$ et $6500 \mathrm{~K}$ et un bon rendu des couleurs (Indice de Rendu de Couleur, IRC $\geq 80$ ).

Pour obtenir de la lumière perçue comme «blanche », il faut au minimum disposer d'un émetteur qui permette une excitation équilibrée des photo-récepteurs (plus précisément, les trois familles de cônes présentant chacune une sensibilité maximale centrée autour du rouge, vert et bleu) présents dans la fovéa de l'œil humain ${ }^{8}$. Ainsi, la lumière « blanche» recherchée peut être obtenue :

- $\quad$ soit par un rayonnement dont le spectre couvre toute la plage visible du spectre (c'est le cas du soleil ou d'un corps incandescent),

- $\quad$ soit, grâce à la physiologie de notre système visuel, par un mélange de bleu, de vert et de rouge (c'est le cas de la télévision couleur), soit encore par un mélange de bleu et de jaune (ceci est lié au fait que les photorécepteurs de notre œil répondent à une couleur et sa complémentaire, ainsi une excitation équilibrée entre ces deux couleurs donne l'impression d'une lumière blanche).

\footnotetext{
${ }^{8}$ La fovéa est la zone de la rétine située dans l'axe de la vision, cette zone est très limitée en surface mais, elle présente la plus forte concentration des photo-récpteurs (essentiellement des cônes) et elle est responsable de la vision « précise » et de la vison « colorée ».
} 
On peut lier le rayonnement du soleil ou celui de l'incandescence, au rayonnement du «corps noir». Ils jouissent de propriétés voisines, en particulier dans leur dépendance vis-à-vis de la température du corps émissif, et c'est pourquoi nous prendrons le «corps noir » comme point de départ.

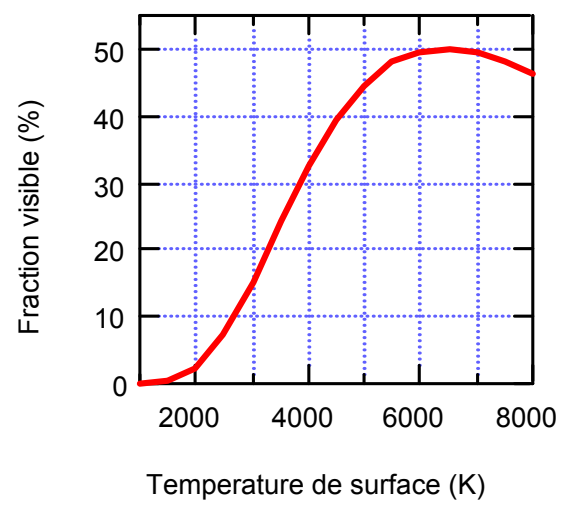

Fig. 5 : Le "poids" du spectre visible dans l'émission totale du corps noir en fonction de la température de sa surface

À la température $T_{C}$, le spectre du rayonnement du corps noir présente, à la longueur d'onde $\lambda$, une luminance spectrique $L_{C N}\left(\lambda, T_{C}\right)$ donnée par la relation de Planck et qui constitue la limite supérieure absolue pour la rayonnement émis autour de cette longueur d'onde. En intégrant la relation de Planck, on peut facilement en déduire la fraction du rayonnement correspondant à la partie visible du spectre. Ainsi, la figure 5 montre le "poids" du spectre visible dans l'émission totale du corps noir en fonction de la température de sa surface. Nous constatons que cette fraction ne peut en aucun cas dépasser la moitié de la puissance rayonnée totale. Ceci est obtenu pour une température de surface de $6500 \mathrm{~K}$ environ. Cependant, le "corps noir" n'est qu'un concept théorique. En réalité, la luminance $L\left(T_{C}, \lambda\right)$ d'un corps réel, lorsqu'elle peut être caractérisée par une température unique, est liée à l'émission du corps noir par une grandeur, l'émissivité, toujours plus petite que l'unité, qui est en particulier une fonction de la température $T_{C}$ caractéristique $\mathrm{du}$ corps et de la longueur d'onde $\lambda$ considérée, $\varepsilon\left(T_{C}, \lambda\right)$, nous parlerons des « corps gris » et nous écrirons:

$$
L\left(T_{C}, \lambda\right)=\varepsilon\left(T_{C}, \lambda\right) L_{C N}\left(T_{C}, \lambda\right)
$$

Ainsi, pour une lampe à incandescence, la quantité de lumière émise dans l'étroite bande spectrale visible dépend principalement de la température du radiateur. Le filament de la lampe est en métal et il est chauffé par effet Joule lors du passage d'un courant électrique. La température maximale est imposée alors par le point de fusion de fusion du métal utilisé. Le tungstène est le métal qui présente le point de fusion le plus élevé à $3410{ }^{\circ} \mathrm{C}$ et il est utilisé à cet effet dans la fabrication des lampes. En pratique, les impératifs mécaniques et thermodynamiques limitent la température à $2700-3000^{\circ} \mathrm{C}$. À cette température la loi de Wien dit que la majeure partie du rayonnement émis par le filament se situe dans la partie infrarouge du spectre $(\lambda>780 \mathrm{~nm})$. Dans ces conditions, on trouve que moins de $25 \%$ du rayonnement total est émis dans la région visible. Ce qui nous conduit à une efficacité énergétique globale de l'ordre de $10 \%$ pour une efficacité lumineuse de l'ordre de $14 \mathrm{~lm} / \mathrm{W}$. Il nous semble bien difficile de faire mieux avec un corps incandescent, sauf, peut-être, en remplaçant le filament métallique par un semi-conducteur (quelques essais ont été réalisés, mais le résultat est loin d'être exploitable...). Mais, comme nous l'avons déjà dit, l'émission d'un spectre continue n'est pas la seule solution pour obtenir de la lumière blanche.

Il semble que le meilleur compromis entre efficacité et qualité consiste à utiliser trois émissions, du bleu, du vert et du rouge, situées au maximum de sensibilité respectifs des trois familles de cônes qui tapissent la fovéa de notre oeil. La figure 6 illustre le principe d'une source qui émettrait uniquement ces trois longueurs d'onde comme un corps noir à $3000 \mathrm{~K}^{9}$.

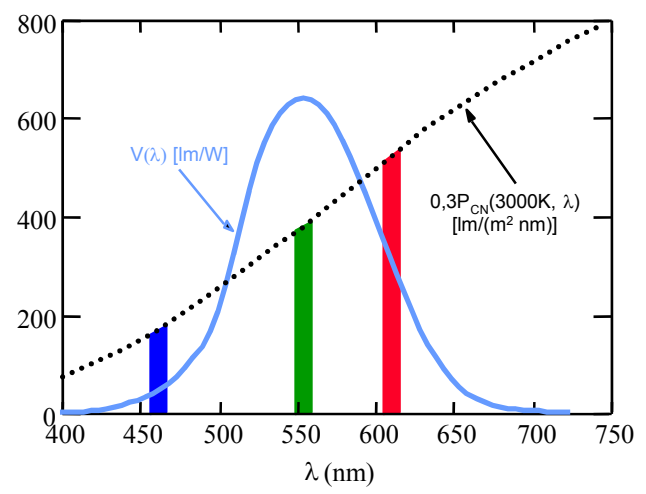

Fig. 6 : On peut obtenir de la lumière blanche en combinant trois photons (bleu, vert et rouge). Une lampe capable d'émettre ces trois photons comme un corps noir à $3000 \mathrm{~K}$ (courbe noire pointillée) aurait une efficacité lumineuse de $300 \mathrm{~lm} / \mathrm{W}$

Dans ce contexte, l'efficacité radiative peut être calculée comme suit :

$$
\eta_{\text {ray }}^{\max }=\frac{\kappa \sum_{i=1}^{3} P_{C N ; i}\left(T_{C}=3000 K, \lambda_{i}\right) V\left(\lambda_{i}\right)}{P_{i n}} \quad \lambda_{i}=\left\{\begin{array}{l}
420 \mathrm{~nm}(\text { bleu }) \\
555 \mathrm{~nm}(\text { vert }) \\
610 \mathrm{~nm} \text { (rouge) }
\end{array}\right.
$$

$P_{C N ; i}\left(T_{C}, \lambda_{i}\right)$ représente la puissance de chacun de trois photons selon la loi de Planck. Une application numérique conduit à $300 \mathrm{~lm} / \mathrm{W}^{10}$. Cette valeur est pratiquement trois fois supérieure à la meilleure efficacité que l'on sache réaliser aujourd'hui. Il nous semble donc réaliste d'affirmer qu'il serait possible, en tenant compte d'un rendement de conversion global du système de l'ordre de $65 \%$, de viser des efficacités lumineuses de l'ordre de $200 \mathrm{~lm} / \mathrm{W}$. Le challenge est de taille et les économies d'énergie associées (à flux lumineux constant) en sont proportionnelles !

Quels sont les challenges pour chaque famille des sources de lumière?

Comme nous l'avons déjà expliqué, l'évolution de la famille «incandescence» semble être compromise, d'ailleurs, un grand constructeur de lampes à déclaré en

\footnotetext{
${ }^{9}$ Cette lampe, malgré son efficacité, aurait une très mauvais rendu de couleur à cause de son spectre quasiment « peu garni »...

${ }^{10}$ Cette valeur est l'efficacité lumineuse concernant la conversion de l'électricité en lumière blanche, elle n'inclus pas les pertes liées au système global (essentiellement pertes dans le circuit d'alimentation de la lampe).
} 
grande pompe qu'il arrêterait la production de ces composants d'ici une dizaine d'années.

En ce qui concerne les lampes à décharge haute pression. Comme on pouvait s'y attendre, du fait des hautes températures atteintes dans ces lampes, leur bilan énergétique indique des pertes thermiques qui représentent 25 à $50 \%$ de la puissance électrique injectée dans la lampe. La diminution des pertes thermiques est donc un objectif primordial dans la recherche d'une efficacité lumineuse améliorée. Les pertes thermiques étant proportionnelles au gradient de température et au coefficient de transfert thermique, ces deux pistes sont à explorer. Il faut cependant noter que les phénomènes de transport de matière jouent un rôle souvent important et que leur maîtrise est nécessaire. La découverte d'enceintes thermiquement plus résistantes permettrait également de diminuer le gradient de température tout en facilitant l'évaporation de certains composés peu volatils. Les lampes à halogénures métalliques céramique (CMHL) vont en partie dans ce sens. Ces lampes produisent de la lumière blanche de bonne qualité (IRC de l'ordre de 95) et elles ont une bonne efficacité (pouvant dépasser $100 \mathrm{~lm} / \mathrm{W}$ ). Elles ont une longue durée de vie (plus de 12000 h) et une excellente stabilité chromatique. Elles sont compactes et elles sont disponibles en plusieurs puissances nominales allant de $20 \mathrm{~W}$ à $400 \mathrm{~W}$. Si les versions de fortes puissances (à partir de $150 \mathrm{~W}$ ) sont en train de conquérir le marché de l'éclairage urbain, celles de faibles puissances s'attaqueront bientôt au marché de l'éclairage intérieur et décoratif en remplaçant les lampes à incandescence.

Plusieurs challenges restent aussi à relever dans le domaine des lampes à décharge basse pression :

- Comment éviter $50 \%$ de pertes dues aux luminophores? La réponse consiste sans doute en l'utilisation de luminophores capables de produire 2 photons visibles pour chaque photon UV incident. Cette solution est plausible mais encore très chère et peu efficace. Une autre solution consisterait à produire directement de la lumière visible en utilisant des substances moléculaires facilement vaporisables ou d'autres gaz. La recherche de nouveaux corps émissifs rejoint le second défi mentionné dans le cas des lampes haute pression.

- Comment éviter l'utilisation de mercure? Le mercure, liquide à la température ambiante est facilement vaporisable; il présente aussi des propriétés atomiques intéressantes et semble être incontournable. Mais, le mercure produit un rayonnement ultraviolet dur, particulièrement à basse pression; il est considéré comme un élément hautement toxique. Plusieurs possibilités peuvent être envisagées pour son remplacement:

- Le xénon produit un rayonnement UV « dur »à $147 \mathrm{~nm}$ qui détruit les luminophores actuels et l'efficacité de production reste faible (presque 2,5 fois inférieure à celle du mercure). Toutefois, la décharge basse pression au xénon produit de la lumière instantanément, tandis que les lampes classiques utilisant le mercure nécessitent un temps de mise en régime correspondant à la vaporisation de ce dernier sous l'action de la chaleur produite par la décharge.
- Le néon, l'un des premiers gaz utilisés dans le domaine des décharges, produit à basse pression $(100 \mathrm{~Pa})$ de la lumière rouge. En augmentant la pression à plus de 104 $\mathrm{Pa}$ et en utilisant des luminophores adaptés, on arrive à produire de la lumière blanche, mais avec une faible efficacité $(20 \mathrm{~lm} / \mathrm{W})$.

- Les émetteurs moléculaires. Malheureusement, la présence des électrodes métalliques restreint dramatiquement le choix des gaz utilisables. Quelques essais ont montré que l'hydroxyle $(\mathrm{OH}-)$ pourrait jouer le rôle du gaz émetteur mais son utilisation est très complexe.

- Comment miniaturiser les lampes fluorescentes? Bien que les CFL soient plus « compactes » que les lampes fluorescentes linéiques, les efforts dans la direction de la miniaturisation de la source continuent sans relâche.

- Comment contrôler la couleur? Le contrôle de la couleur des tubes basse pression est d'une importance capitale pour des applications comme la signalisation et l'affichage publicitaire. Il est possible de contrôler la couleur de la décharge en choisissant convenablement le mélange gazeux (avec ou sans mercure) et les luminophores associés. Une autre méthode, plus souple, consiste à contrôler l'excitation sélective des corps émissifs en changeant les caractéristiques de l'alimentation électrique de la décharge (forme d'onde, rapport cyclique, fréquence). Ainsi, une lampe $\mathrm{Hg}-\mathrm{Ne}$ peut basculer de la couleur bleue $(\mathrm{Hg})$ avec une excitation sinusoïdale, au rouge $(\mathrm{Ne})$ lorsque celle-ci est soumise à un mode impulsionnel.

- Comment réduire le temps de mise en régime ? Signalons pour commencer que cette notion de temps de mise en régime est délicate ; nous sommes en effet confrontés à des écarts importants suivant le mode de mesure. Les données dont nous disposons nous permettent de parler d'un délai de l'ordre de $10 \mathrm{~min}$ alors que des mesures réalisées par d'autres donnent plutôt 3 min... Il ne nous est pas possible de trancher, mais ce que nous devons préciser ici, c'est que ce problème de la mise régime est primordial pour l'utilisateur des lampes CFL qui, habitué aux lampes à incandescence, veut un allumage instantané. Le développement des ballasts électroniques robustes pourrait résoudre partiellement ce problème.

Presque toutes les lampes à décharge utilisées pour l'éclairage sont dotées d'électrodes pour injecter l'énergie électrique dans la décharge et assurer la transition de la conduction métallique à la conduction gazeuse. Bien qu'aujourd'hui la technologie des électrodes soit mieux maîtrisée, la présence de ces éléments métalliques dans le plasma limite sérieusement la durée de vie de la lampe et restreint également le choix des gaz émetteurs. La suppression des électrodes résout plusieurs problèmes et simplifie par ailleurs la réalisation des lampes. Il est tout à fait possible d'injecter la puissance électrique dans une décharge en couplant, capacitivement ou inductivement, le plasma avec le circuit d'alimentation. L'utilisation de microondes est aussi une alternative. Nous rencontrons aujourd'hui de trois technologies différentes : les lampes fluorescentes dites «à induction", les lampes à barrière diélectrique dites «à excimères » et les lampes à micro- 
ondes. Les lampes à barrière diélectrique utilisent l'émission des molécules excimères. Ces molécules qui n'existent pas dans les conditions habituelles (le niveau fondamental est « dissociatif ») peuvent exister pendant des temps très courts à un état excité radiatif. Aujourd'hui nous maîtrisons bien la production de ces molécules instables dans une décharge électrique hors d'équilibre à la pression atmosphérique. Les lampes à excimères ont un avantage: en l'absence des électrodes toute forme géométrique du tube devient possible, la durée de vie, comme dans le cas des lampes à induction, est très longue. Par ailleurs, nous avons un très large choix de longueur d'onde car nous ne sommes plus limités par les électrodes au choix du corps émissif. Ce type de lampe peut être considéré comme une révolution dans le domaine des sources de lumière. Nous sommes aujourd'hui persuadés que l'utilisation des émissions moléculaires combinées avec la technologie des lampes sans électrodes nous mènerons d'ici quelques années à la création des nouveau produits bien plus efficaces, produisant une lumière de meilleure qualité et en forte quantité, avec une longue durée de vie. S'agit-il de la source de lumière du future?

Les LEDs véhiculent beaucoup d'espoirs pour l'éclairage. Dans un «papier blanc » commandé par le Sénat des Etats-Unis, R. Haitz et all ont élaboré 2 scénarii [5]: le premier, simplement «évolutionnaire » prévoit pour 2010 une diode blanche de $50 \mathrm{~lm} / \mathrm{W}$ tandis que, le deuxième, dit " révolutionnaire", vise un dispositif de $200 \mathrm{~lm} / \mathrm{W}$ d'ici 2025. Selon le premier scénario, les $50 \mathrm{~lm} / \mathrm{W}$ semblent être un objectif réaliste, que l'on peut atteindre sans effort considérable. Dans ce contexte, les LEDs peuvent très bien remplacer d'ici 2025, 20 à $30 \%$ des lampes à incandescence et halogènes. Ceci entraînerait une économie d'énergie de l'ordre de $400 \mathrm{TWh} / \mathrm{an}$ (correspondant à une réduction de capacité de production de $50 \mathrm{GW}$ ) ; à titre de comparaison la production Française annuelle de l'électricité est de l'ordre de 550-600 TWh . Dans le deuxième scénario, atteindre une efficacité de $200 \mathrm{~lm} / \mathrm{W}$ nécessite clairement un effort considérable. Dans ce cas, les LEDs seraient susceptibles de remplacer les lampes fluorescentes et pourraient représenter plus de $50 \%$ de parts du marché des sources de lumière d'ici 2025-2030. L'économie d'énergie escomptée serait de l'ordre de $1000 \mathrm{TWh} / \mathrm{an}$ (correspondant à une réduction de capacité de production de $120 \mathrm{GW}$ ). Aujourd'hui, selon Russ Arensman [6] le marché mondial des LEDs pèse presque 3 milliards d'euros et son taux de croissance annuel est de $14 \%$. Si ce rythme se confirme et se maintient pendant les 5 années à suivre, ce segment du marché représenterait en 2009 pas loin de 6 milliards d'euros pour dépasser les 10 milliards dans la décennie à suivre. Cependant, aujourd'hui la pénétration des LEDs dans le domaine de l'éclairage général reste de l'ordre de 5\% (figure 7).

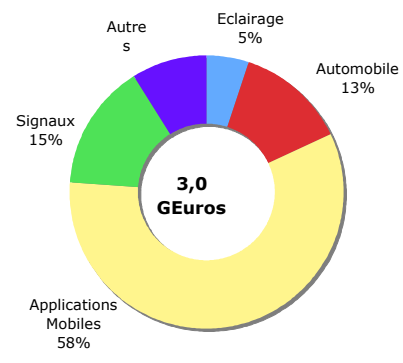

Fig. 7: Marché des LEDs en 2004

Cette part augmentera-il dans les années à suivre? Malgré l'optimisme général soutenu par des avancées scientifiques spectaculaires reprises en fanfare par les fabricants, cette question demeure aujourd'hui sans réponse claire. Les LEDs présentent des avantages majeurs :

- fort potentiel de développement de leur efficacité (100\% théorique de transformation de l'électricité en lumière).

- grande durée de vie: l'objectif annoncé, et qui fait consensus, est d'obtenir une diode d'une durée de vie de 40000 à 50000 heures d'ici 2025 (plutôt que les 100000 heures espérées il y a quelques années),

- couleurs saturées de bonne qualité, assurant une bonne perception de la lumière par l'utilisateur,

- alimentation aisée, faible demande en énergie (bien qu'une stabilisation extrême est nécessaire pour un bon contrôle de la couleur),

- légèreté.

Mais il existe également des inconvénients, bien connus, eux aussi. Les points qui posent actuellement problème sont les suivants :

- Un des problèmes majeurs des LEDs est que la lumière émise est toujours monochromatique. Du coup, une des problématiques essentielle pour la recherche consiste à travailler sur l'obtention d'une «LED blanche» ayant une efficacité de plus de $100 \mathrm{~lm} / \mathrm{W}$ tandis qu'aujourd'hui les jonctions commerçables présentent une efficacité de $25 \mathrm{~lm} / \mathrm{W}$.

- Le schéma précédent montre clairement que les LED ne peuvent produire que des «plumes» de lumières : la lumière est à la fois directionnelle et monochromatique. C'est évidemment un énorme défaut pour l'éclairage général (où l'on a besoin d'une lumière diffuse et non éblouissante). Pour corriger ce problème il faut une optique lourde, chère et qui nuit à l'efficacité lumineuse. Cette caractéristique cantonne par ailleurs actuellement les applications aux spots d'éclairage et à l'éclairage d'appoint.

- Le vieillissement est erratique, la couleur « dérive avec le temps ». Le flux produit par les LED blanches diminue presque linéairement dès la première heure de fonctionnement. Les mesures de la chute du flux ont été réalisées sur une diode blanche de $1 \mathrm{~W}$ montrent que le flux diminue de moitié en 6000 heures (données 2000, on ne fait guère mieux aujourd'hui...). Des progrès dans ce domaine sont donc également nécessaires pour que les diodes électroluminescentes puissent, un jour, dominer le marché de l'éclairage. 
- Il existe des problèmes de reproductibilité de production en grande série: pour la LED blanche uniquement (température de couleur non identique).

- Les LED sont limitées à de faibles puissances : inférieures à $5 \mathrm{~W}$, actuellement. Il s'agit essentiellement d'un problème de management thermique de la jonction car la chaleur engendrée par le fonctionnement de la diode modifie ses performances.

- La consommation énergétique est faible. Cette constatation contribue à lui donner une image « écologique », mais, la technique de production, elle, est moins «verte » (industrie du semi-conducteur)... Il faut situer ce point par rapport à l'industrie des lampes actuelle qui est très propre (peu de produits toxiques générés).

Ces problèmes que nous signalons ici sont un instantané, réalisée en 2006 et feront peut-être sourire dans quelques années, mais aujourd'hui personne ne peut dire à quelle vitesse ils seront résolus.

Les perspectives de recherche et développement dans le domaine des LEDs sont énormes, la liste suivante indique quelques-unes jugées pertinentes :

- Recherche fondamentale sur les matériaux et la génération de la lumière : il faut tout d'abord mieux comprendre les mécanismes de génération de lumière par les semi-conducteurs. Pour cela il faut développer de nouvelles techniques de diagnostic adaptées aux LED et des modèles mathématiques plus sophistiqués que ceux dont nous disposons aujourd'hui. Dans l'immédiat, la compréhension des problèmes liés aux défauts de dopage de type $\mathrm{p}$ et à l'incorporation de fortes concentrations d'Indium et d'Aluminium dans les diodes vertes InGaN et AIGalnN, nous conduisent à la création de diodes vertes plus efficaces. L'étude du confinement des porteurs dans les LED à base de phosphides semble, quand à elle, être la clef pour la réalisation de diodes rouges beaucoup plus efficaces.

- Matériaux pour les substrats : aujourd'hui pour la création des diodes de la famille GaN, qui semblent être parmi les plus prometteuses, il faut utiliser des substrats en Saphir, $\mathrm{SiC}$ ou bien $\mathrm{GaN}$. Chacun de ces trois matériaux présente des avantages et des inconvénients. Toutefois, nous ne savons pas produire des grandes surfaces sans défaut avec ces matériaux. La solution de ce problème doit être considérée comme une priorité dans ce domaine. Par ailleurs il serait souhaitable de pouvoir diminuer le coût de production du substrat à moins de $70 € / \mathrm{cm}^{2}$ (pour les diodes UV, vertes et bleues).

- Technologie des réacteurs pour la création des diodes par épitaxie : les réacteurs utilisés pour la production en série des diodes électroluminescentes de la famille GaN ne sont que des machines créées pour la production des diodes en GaAs. Si leur fiabilité est bonne pour la production des diodes en GaAs, elle est déplorable pour la réalisation des diodes modernes en GaN. Ainsi, les diodes produites en série par ces machines présentent quasi-systématiquement des efficacités 1,5-2 fois inférieures à celles constatées sur les prototypes. Par ailleurs, dans la même série produite par une machine, on peut constater d'importantes dispersions de caractéristiques. L'exemple caricatural est le tri manuel en fonction de la « couleur» effectué à la fin de la ligne de production des diodes blanches de forte intensité... Pour avancer dans ce domaine, il vaut mieux comprendre la chimie du process et les mécanismes de croissance des nitrides afin d'optimiser les réacteurs.

- Luminophores : les problèmes liés à la faible absorption de la lumière bleue par les phosphores jaunes ont déjà été évoqués. Dans un premier temps, il convient de développer des luminophores capables d'absorber et de convertir efficacement des photons de longueurs d'onde comprises entre 370 et $470 \mathrm{~nm}$, dans un deuxième temps il sera nécessaire d'étudier d'autres combinaisons possibles de couleurs complémentaires et de développer des phosphores adéquats. Par ailleurs, comme pour les lampes fluorescentes, le développement des «quantumsplitting phosphors » marquera une étape décisive pour les LED.

- Extraction de la lumière et packaging : l'amélioration des caractéristiques optiques des substrats et contacts transparents, des couches réfléchissantes,... constitue un axe de recherche important dans un futur proche. Améliorer la géométrie des jonctions afin d'augmenter l'efficacité de l'extraction de la lumière, ou même faire appel à des structures complexes comme les cristaux photoniques sont des challenges à plus long terme. Enfin, il ne faut pas oublier que la jonction n'est qu'une petite partie de la diode, et que le packaging joue un rôle primordial pour son efficacité et sa fiabilité. Des challenges d'ordre technologique sont à prévoir dans ce domaine. Par exemple, pour une diode de forte intensité, il faudrait un matériau transparent et facilement usinable ayant un indice de réfraction supérieur à 1,6 et une transmittance de $80 \%$ de 440 à $650 \mathrm{~nm}$ susceptible de bloquer complètement des photons courts $(\lambda<440 \mathrm{~nm})$. Ce matériau doit conserver ces caractéristiques pendant au moins 50000 heures tout en étant soumis à une température de $150{ }^{\circ} \mathrm{C}$. Enfin, il faut que la concentration d'eau dans ce matériau reste de l'ordre de quelques ppm et que ces caractéristiques mécaniques soient compatibles avec l'application envisagée...

- Luminaires et électronique : pour que les LED puissent pénétrer le marché de l'éclairage, il faut développer des luminaires spécifiques qui prennent en compte la forte directivité de la lumière, les faibles dimensions de la source de lumière et surtout sa sensibilité à la température, humidité et tout autre facteur corrosif rencontré dans son environnement. Enfin, compte tenu $\mathrm{du}$ fait que la production d'une quantité de lumière suffisante pour l'éclairage général implique un nombre important de LED, une électronique de commande sophistiquée est nécessaire. Notons ici que la couleur d'une LED est très sensible à la fois à la tension et au courant de l'alimentation. Une faible variation sur l'une ou l'autre de ces grandeurs provoque une importante dérive de la couleur. Par ailleurs, le comportement de chaque diode se modifie avec le temps (le flux baisse, la couleur dérive) plus ou moins lentement, mais, à cause des lacunes au niveau de la production (voir 
précédemment) cette variation est différente d'une diode à l'autre. Il faut donc les contrôler une à une...

Ces objectifs annoncés clairement par les chercheurs pour les 15 années à venir constituent des paris ambitieux. Sont-ils réalistes ? Répondre à cette question par «oui » ou par «non» reviendrait à spéculer sur la forme d'un puzzle auquel manquent des pièces maîtresses !

Dans cette famille, une autre révolution se prépare : Les diodes électroluminescentes organiques (O-LEDs) sont la variante organique des LEDs. Cette technologie brevetée en 1987 par société Kodak a été initialement destinée aux applications d'affichage et sa première application commerciale est apparue vers 1997. Depuis les O-LEDs ont énormément évolué, au point d'obtenir des modules produisant de la lumière blanche avec une efficacité de 32 $\mathrm{lm} / \mathrm{W}$. Dans ce contexte, et, si les problèmes de durée de vie se résolvent les O-LEDs deviendront un concurrent sérieux des LEDs classiques car à l'encontre des LEDs les O-LEDs ont une «étendue» importante nécessaire pour les applications d'éclairage général. En effet, dans un module OLED chaque diode, dont l'épaisseur ne dépasse pas le millimètre, est composée de trois couches d'un semiconducteur organique (film plastique) entourées par des électrodes transparentes (au moins une des deux si l'on veut la lumière puisse « sortir »). Chaque « pixel de lumière » est constitué de trois diodes électroluminescentes juxtaposées (une rouge, une verte et une bleue). En voici quelques autres avantages de O-LED qui plaident en leur faveur :

- Mise en œuvre en surface importante possible (en panneau éclairant), ce qui implique un éclairage important possible et des économies d'échelle associées.

- Efficacité potentielle importante : 20-40 lumen/W (au stade de prototype).

- Alimentation simple (secteur redressé, 10V); Gradation lumineuse possible, théoriquement sans problème.

- Lumière non-directionnelle (émetteurs « lambertiens » à grande étendue), colorée ou blanche.

- Produits légers et souples (plastique).

Le futur montrera si cette technologie émergente prendra des parts de marché dans le domaine de l'éclairage.

\section{SOURCES DE LUMIERE ET DEVELOPPEMENT DURABLE}

Pour mieux comprendre l'importance des sources de lumière artificielle au développement durable il faut commencer par la définition de ce dernier. Ainsi, l'expression «développement durable» désigne «un développement qui permet de répondre aux besoins présents sans compromettre la capacité des générations futures à faire face aux leurs ». En ce qui concerne la production de la lumière ces lignes peuvent prendre un sens différent pour les pays occidentaux et pour les pays en voie de développement.

La consommation énergétique d'un pays occidental pour l'éclairage varie entre $5 \%$ et $15 \%$ de sa propre production énergétique (avec un cas singulier: les USA, où ce pourcentage atteint 19-20\%). Sachant qu'approximativement $60 \%$ de cette quantité d'énergie est utilisée pour l'éclairage de bâtiments à destination industrielle et tertiaire il est possible de contenir la croissance de la demande en utilisant des moyens légaux (normes et directives) qui imposent de facto l'utilisation des sources de lumière énergétiquement efficaces (3). L'éclairage du secteur résidentiel, quant à lui, représente $30 \%$ de la note globale d'énergie et il est bien plus difficile à réglementer. La solution consisterait à user des moyens plutôt incitatifs et surtout renseigner la population des avantages des certaines technologies économes en énergie. En Europe, par exemple, $\mathrm{si}$, dans nos maisons, nous pouvions remplacer une lampe à incandescence de $75 \mathrm{~W}$ par une lampe fluo compacte (lampe «basse consommation ») de $15 \mathrm{~W}$ qui produit la même quantité de lumière nous pouvions réaliser des économies considérables. Chaque foyer économiserait en moyen 150 $\mathrm{kWh}$ par an et compte tenu que l'Europe compte, en gros, 150 millions des foyers, les économies annuelles monteraient à plus de $22 \mathrm{TWh}$ ou l'équivalent de 4 millions de tonnes pétrole (TEP).

Il faut aussi insister grandement au fait que « éclairer » ne signifie point «inonder» un objet ou un espace de lumière ! Cependant, aujourd'hui dans les pays riches nous aurions plutôt tendance à augmenter la quantité de la lumière sous prétexte que la performance visuelle augmente. On sait pourtant que la majorité des êtres humains se dit satisfaite du niveau d'éclairement à partir de 400-500 lux, sans pouvoir être capable de distinguer une amélioration des conditions de travail ou de la qualité de vie au-delà de ces valeurs...

En ce qui concerne maintenant les pays en voie de développement la situation est différente. L'éclairage représente aujourd'hui la majeure partie de leur consommation électrique (voire §II). L'éclairage domestique est ici prédominant. Cette situation s'explique facilement compte tenu que la lumière est un besoin intime de l'Homme, et, bien entendu, dès que l'électrification avance la population en profite en installant tout d'abord des lampes. Par ailleurs, dans ces pays, le coût de la lampe est un facteur majeur pour le choix du type de la source de lumière. Ainsi les lampes à incandescences dominent le marché car les lampes basse consommation sont souvent inabordables. Comme il s'agit d'un usage principalement domestique, il est difficile d'imposer le type de lampe par décret (sauf dans certains pays au régime autoritaire, comme Cuba, que les lampes à incandescence ne sont plus importées...). Par ailleurs il est difficile pour l'économie de ces pays de supporter à long terme des mesures incitatives conséquentes. L'équation semble difficile à résoudre, pourtant il existe une solution. Il faut développer des produits basés sur des technologies actuelles (lampes fluo compactes) plus « rustiques » mais bien plus robustes et surtout moins chers. La robustesse du produit est ici primordiale car qualité du réseau électrique peut être plus aléatoire que dans des pays occidentaux et les lampes à décharge supportent moins bien les fluctuations de la tension. Par ailleurs, la robustesse garantie une faible fréquence de maintenance. Toutefois, ces produits adaptés et moins chers doivent obéir aux mêmes exigences énergétiques et environnementales que les produits destinés aux marchés occidentaux. Évidemment le développement d'un tel produit nécessite un investissement initial qui ne peut être supporté par les pays riches ou les groupes industriels du secteur. Toutefois, le retour à l'investissement est rapide et profitable pour tous. Il faut enfin doter ces pays des moyens de qualification de produits qui pénètrent leur matché afin d'éviter des supercheries. 
Certains ne prônent aujourd'hui qu'une solution pour ces pays serait l'utilisation extensive des sources d'énergie renouvelables (surtout le soleil) pour alimenter des lampes économes (fluo compactes, LEDs...). La solution semble attractive, mais elle ne résiste pas à une analyse critique. L'utilisation de l'énergie solaire (disponible par définition aux heures où l'éclairage artificiel n'est pas indispensable) nécessite des unités de stockage de l'énergie. Ces unités sont aujourd'hui coûteuses et volumineuses, elles ont une duré de vie courte et elles nécessitent un entretien régulier par des spécialistes. Enfin, elles contiennent beaucoup des matières nocives pour l'environnement au point de nécessiter d'un recyclage à la fin de leur vie. Bref, elles sont incompatibles avec la définition même du développement durable pour le pays en voie de développement.

Quelques cas particuliers existent aussi: les pays producteurs de pétrole en est un. Dans un pays comme le Koweït où l'énergie est subventionnée à la hauteur de $80 \%$ par l'état, le coût du kilowatt-heure est si faible pour la population dont l'achat d'une lampe économique ne se justifie pas. Une question se pose : comment faire des économies d'énergie dans ce cas? Une solution étudiée par le gouvernement consiste à acheter un grand nombre des lampes fluo compactes et les distribuer gratuitement à la population. Un calcul de retour à l'investissement basé sur le remplacement de la moitié de lampes d'un foyer, montre que l'investissement initial est amorti en 9-10 mois. Toutefois, une étude a montré que $15 \%$ de la population refuserait d'utiliser ces lampes car elles sont considérées comme « difformes »... Cela montre que la population ne connaît pas les nouvelles générations du produit qui ressemble de près à certaines lampes à incandescence. Ici le développement durable passerait par une campagne d'information et « d'éducation » de la population.

Il existe enfin en 2004 sur la terre plus d' 1,7 milliards de personnes que n'ont pas accès à l'éclairage électrique (ils étaient 2 milliards en 1996, selon la banque Mondiale) [7].

Cette fraction de la population continue à utiliser des lampes à pétrole pour s'éclairer. Il se trouve que pour ces populations même le prix du pétrole pour l'éclairage est un fardeau. Pour la même quantité de lumière produite, les dépenses liées à l'éclairage par ce biais sont 325 fois plus importantes que celles qui sont engendrées par une lampe à incandescence ou même 1625 fois supérieures en les comparant à une lampe fluo compacte. La dépense annuelle moyenne de ces populations pour l'éclairage avec du pétrole est de l'ordre de 100 euros par foyer pour produire l'équivalent de 12000 lumen-heures par an. Pour une maison électrifiée, la même quantité de lumière est produite par une lampe à incandescence de $100 \mathrm{~W}$ en $10 \mathrm{~h}$. Globalement, pour une dépense équivalente, ces populations disposent seulement $1 \%$ de la quantité de lumière d'un pays électrifié... Donner accès à l'éclairage électrique à ces populations est un facteur de développement durable. Ceci ne veut pas forcément dire qu'il faut connecter tout point du globe au réseau électrique. La fondation «Light-up the world» procure, pour un prix modique (1 US\$), à des populations déshérités des petites lampes frontales utilisant des LEDs blanches d'une puissance globale de $1 \mathrm{~W}$ connectées à une pile ${ }^{11}$. Cet éclairage d'appoint permet aux personnes de prolonger leurs activités d'artisanat dans la soirée et augmenter ainsi le revenue familial, mais elle permet surtout aux enfants de pouvoir étudier et suivre ainsi une scolarisation normale.

\section{EN GUISE DE CONCLUSION}

Nous avons aujourd'hui la certitude que les besoins en éclairage de l'Humanité ne feront que croître ; on pourrait s'attendre à un facteur de l'ordre de 2 pour les deux décennies à venir. Comment satisfaire cette croissance sans que la demande énergétique du secteur progresse dans la même proportion? Il s'agit ici d'une question qui relève à $100 \%$ du développement durable. La figure 8 illustre de façon très synoptique des moyens que nous devons mettre en œuvre pour répondre à cette question.

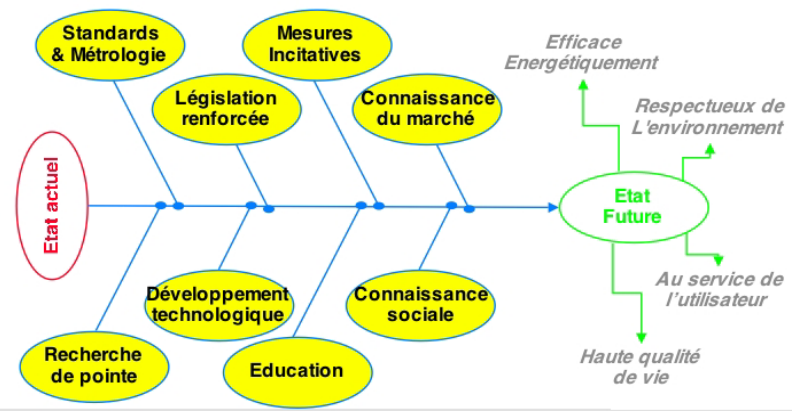

Fig. 8 : Comment satisfaire les besoins futurs en éclairage sans compromettre les ressources énergétiques et environnementales

Le système d'éclairage future issu de ce processus doit satisfaire bien sur les besoins en lumière, mais il doit respecter les ressources énergétiques et environnementales, il doit contribuer à la qualité de vie de l'Homme et enfin il doit être au service de l'utilisateur. Les qualités demandées touchent de plus en plus à l'aspect chromatique des sources, à leur durée de vie et à leur stabilité (chromatique en particulier) ainsi qu'à des conditions d'emploi adaptées (allumage et ré-allumage instantanés, dimensions). Les réponses sont sans doute d'une part une meilleure connaissance de la chimie et des mouvements de fluides dans la source, d'autre part dans la maîtrise de l'électronique (mode d'alimentation) et de son adaptation à la lampe (électrodes ou couplage) et à la mise en régime. Enfin une meilleure modélisation de l'interaction réseau-source et du vieillissement des lampes pourrait très vraisemblablement diminuer le coût de la gestion (maintenance en particulier) des réseaux urbains d'éclairage comme celle des bâtiments.

Augmenter la durée de vie de la lampe, améliorer son rendu de couleur, miniaturiser la source et le système, supprimer le mercure et autres éléments toxiques sans perte d'efficacité, obtenir la mise en régime instantanée..., voilà bien des objectifs intéressants pour le futur.

\footnotetext{
${ }^{11}$ Fondation Light-up the World, http://www.lightuptheworld.org/
} 


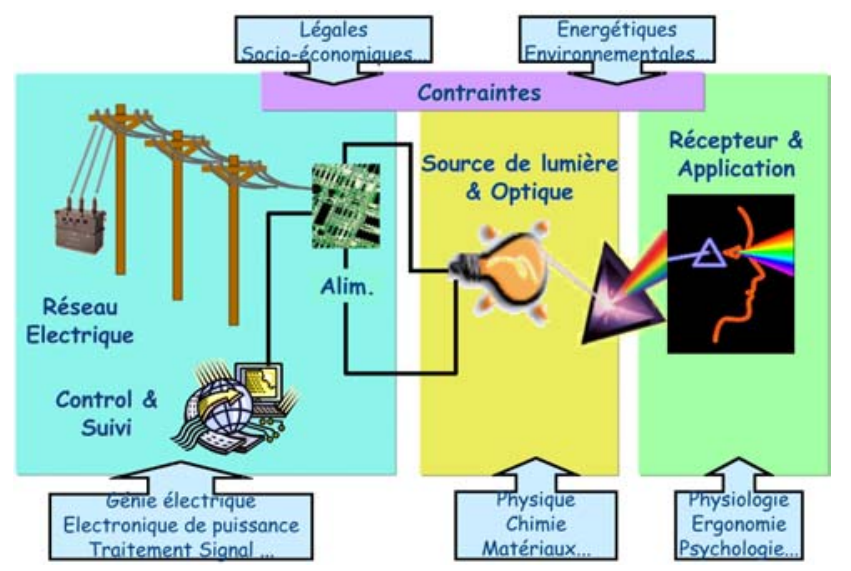

Fig 9 : Système d'éclairage

Cependant comme le montre la figure 9, si l'on veut a réussir le pari d'une meilleure source de lumière, économe qui respecte son environnement et qui contribue à l'amélioration de la qualité et le confort de notre vie, on doit traiter toutes les aspects d'un système complexe et ceci ne peut se faire que par le biais d'une étude vraiment pluridisciplinaire.

\section{REFERENCES}

[1] Données de l'International Association for Energy Efficient Lighting, accessibles sur le cite web http://www.iaeel.org/

[2] P. Mills, Why we're here: The \$230-billion global lighting energy bill, Proc. 5th Right Light Conference, pp. 369-385, Nice (France), May 2002

[3] M. Scholand, J. Brodrick, E. Petrow, Lighting Energy Consumption Trends and Conservation Opportunities in U.S. Buildings, Proc. 1st COST-529 meeting, Toulouse (France), June 2002

[4] European Parliamant and Council, Directive 2002/95/EC-27/01/2003, On the restriction of the use of certain hazardous substances in electrical and electronic equipment

[5] R. Haitz R., Kish F., Tsao J., Nelson J., Another semiconductor revolution: This time it's lighting, Comp. Semiconductor Magazine, March 2002

[6] R. Arensman R., LED market lights up, Reed Business Information, a division of Reed Elsevier Inc, Electronic Business 4/1/2005

[7] R. Jones, Zachery M. Gentry, Alternatives to Fuel-Based Lighting in Rural China, Proc. of the 6th Int. Conf. on Energy-Efficient Lighting, 9-11 mai 2006 Shanghai (Chine), pp 397-400

\section{Biographie de l'auteur}

Georges Zissis est né à Athènes en 1964. En 1986, il a obtenu une Maîtrise de Physique à l'Université de Crète (Grèce) suivi d'un DEA en Physique de Plasmas en 1987 à l'Université Paul Sabatier (Toulouse 3) et d'un Doctorat de l'Université dans la même discipline en 1990. II est spécialiste de la science et technologie des sources de lumière.

II a commencé sa carrière d'Enseignant - Chercheur comme Maître de Conférences à l'Université Paul Sabatier en 1992 et depuis 2005, il est Professeur des Universités dans le même établissement (63e section CNU).

Aujourd'hui il est Directeur Adjoint du Laboratoire Plasma et Conversion d'Energie (LAPLACE), UMR5213 (CNRS, Univ. Paul Sabatier et Institut National Polytechnique de Toulouse).

Il est président du group régional Midi-Pyrénées de l'Association Française de l'Eclairage et Secrétaire National de la même société savante.

II préside depuis 2006 le comité technique « Industrual Lighting and Displays » de l'IEEE IAS.

II a créé et dirigé pendant 5 (2001-2006) ans le réseau Européen COST-529 « Efficient Lighting for the 21st Century ».

II a mis en place en 2006 la Licence Professionnelle "Eclairage Public et Réseaux d'Energie » à St Affrique (Aveyron).

Pour ces travaux sur la normalisation des sources de lumière, il a obtenu en 2006 le 1er prix du Centenaire de la Commission Internationale de l'Electrotechnique (IEC).

II a publié plus de 60 articles et plus de 150 communications dans son domaine. II a dirigé jusqu'à aujourd'hui 7 doctorats de l'université. 